\title{
CCL19-1 A MUCOSAL CHEMOKINE SPECIALIZED IN RAINBOW TROUT NASAL IMMUNITY
}

\author{
Sepahi A ${ }^{1}$, Tacchi L ${ }^{1}$, LaPatra $\mathrm{SE}^{2}$, Salinas $\mathbf{I}^{*}$ \\ ${ }^{1}$ Center of Evolutionary and Theoretical Immunology Department of Biology University of New Mexico, \\ New Mexico, USA. \\ ${ }^{2}$ Research Division Clear Springs Foods Inc, Buhl, Idaho, USA.
}

\begin{abstract}
Chemokines are a large group of small proteins, which have fundamental immunological roles in migration, activation, and differentiation of leukocytes. Chemokines and chemokine receptors rapidly diversified in teleost fish. The chemokine (C-C motif) ligand 19 (CCL19) is known to be crucial for both lymphoid cell trafficking and the structural organization of lymphoid tissues in mammals. We report that CCL19, diversified in salmonids leading to the presence of three CCL19 forms. Previous studies in our laboratory showed $100 \%$ protection in nasally vaccinated trout 7 days post immunization with a live attenuated infectious hematopoietic necrosis virus vaccine and a clear induction of CCL19-1 transcripts on day 4 in the olfactory organ as measured by oligomicroarray. CCL19-1 is unique to salmonids and its sequence most resembles the zebrafish CCL19. Compared to the other CCL19 genes (-2 and -3); we found that CCL19-1 is constitutively expressed in all trout mucosa- associated lymphoid tissue (MALT) at much higher levels than in primary lymphoid tissues. The other two CCL19 forms did not show this expression pattern. In vivo, CCL19-1 expression is highly up-regulated in nasopharynx-associated lymphoid tissues (NALT) following nasal vaccination, whereas CCL19-2 expression is moderately up-regulated and CCL19-3 expression is decreased. Trout recombinant CCL19-1 is not chemotactic in vitro but it modulates the expression of immune genes in trout head-kidney leucocytes such as CCR7, IFN $\gamma$ and CD8 $\alpha$. Similarly, in vivo nasal delivery of rCCL19-1 induces CD8 T cell responses in the local nasal mucosa. These results show that expansion of chemokine genes in salmonids led to acquisition of specialized immune roles for some of these molecules such as CCL19-1, which acquired a key role in trout mucosal immunity.
\end{abstract}

\section{KEYWORDS}

CCL19, NALT, trout, nasal immunity, mucosal immunity

*Corresponding author. Tel.: +1 505-277-0039

E-mail address: isalinas@unm.edu 